\title{
Aleaciones de aluminio amorfas y nanoestructuradas
}

\author{
A. García-Escorial ${ }^{(*)}$, K.R. Cardoso ${ }^{(*)}\left({ }^{* *}\right)$ y F.E. Audebert $\left.{ }^{*}\right)(* * *)$
}

\begin{abstract}
Resumen En este trabajo se recoge la labor desarrollada en el CENIM en aleaciones de base aluminio amorfas y nanoestructuradas. Se presentan los resultados obtenidos hasta ahora en el estudio de aleaciones Al-LTM-ETM, Al-Fe-Nb y Al-Cu-Zr, y Al-LTM-RE, Al-Fe-Nd (LTM = Late Transition Metal, $\mathrm{ETM}=$ Early Transition Metal y RE = Rare Earth), procesadas por solidificación rápida, mediante atomización por gas inerte y temple ultrarrápido sobre rueda, así como por aleado mecánico en molino de bolas de alta energía.
\end{abstract}

Palabras clave: Aleaciones de aluminio. Amorfos. Nanoestructuras.

\section{Amorphous and nanostructured aluminium alloys}

\begin{abstract}
The work carried out in CENIM on amorphous and nanostructured aluminium alloys is described. Results about Al-LTM-ETM, Al-Fe-Nb and Al-Cu-Zr, and Al-LTM-RE, Al-Fe-Nd (LTM = Late Transition Metal, ETM = Early Transition Metal y RE = Rare Earth) alloys obtained by rapid solidification, inert gas atomization and melt-spinning, as well as mechanical alloying are presented.
\end{abstract}

Keywords: Aluminium alloys. Amorphous. Nanostructures.

\section{INTRODUCCIÓN}

Las aleaciones de aluminio, junto con los aceros, son los materiales metálicos más utilizados. Las aleaciones de aluminio irrumpieron en el campo de las aplicaciones a principios de siglo, a partir de la observación del proceso de endurecimiento por precipitación de soluciones sólidas sobresaturadas que dio lugar a los duraluminios, que alcanzan resistencias a la tracción (UTS) de hasta $600 \mathrm{MPa}$. Posteriormente, en la década de los ochenta, se desarrollaron nuevas aleaciones de aluminio con mejores características, como aleaciones amorfas, con UTS de hasta $1.200 \mathrm{MPa}$, y nanocomposites de nanocristales embebidos en una matriz amorfa que alcanzan resistencias a la tracción de hasta $1.550 \mathrm{MPa}$ (1). El desarrollo de estas nuevas aleaciones ha sido posible gracias a las nuevas técnicas disponibles de procesado de no equilibrio, como la solidificación rápida y el aleado mecánico.

(*) Centro Nacional de Investigaciones Metalúrgicas, CENIM (CSIC), Avda. de Gregorio del Amo 8, 28040Madrid (España).

(**) Univ, Federal de Sâo Carlos, Caixa Postal 676, Sâo Carlos (Brasil).

(***) Univ. de Buenos Aires, Paseo Colón, 850. 1063 Buenos Aires (Argentina).
Las aleaciones de aluminio son aleaciones ligeras de alta resistencia específica y de gran interés tecnológico, por lo que se desarrolla un enorme esfuerzo investigador para aumentar su rango de temperaturas de trabajo y su resistencia a la tracción. La solidificación rápida permite introducir más elementos de aleación en las aleaciones de aluminio y refina el tamaño de grano, con lo que se puede mejorar considerablemente su estabilidad térmica y su resistencia a la tracción. Así, las aleaciones microcristalinas $\mathrm{Al}-\mathrm{Cr}-\mathrm{Zr}$ (2) y las $\mathrm{Al}-\mathrm{Fe}-\mathrm{Ce}$ (3) obtenidas por solidificación rápida y consolidadas por extrusión están ya comercializadas. Las aleaciones microcristalinas de base aluminio obtenidas por solidificación rápida se vienen estudiando en el CENIM tradicionalmente (4 y 5).

En 1988, dos grupos de investigación obtuvieron simultáneamente aleaciones amorfas de base aluminio en sistemas Al-LTM-RE (6 y 7), consiguiendo mayores resistencias a la tracción. Más recientemente, Inoue (8) obtuvo mayores resistencias mecánicas al cristalizar parcialmente estas aleaciones, lo que da lugar a cristales nanométricos de aluminio c.c.c, embebidos en una matriz amorfa.

Además, Shechtman, en 1984 (9), observó, en aleaciones de aluminio solidificadas rápidamente, 
la existencia de los cuasicristales y desde entonces la mayoría de estructuras de este tipo se han observado en aleaciones de aluminio. Por tanto, el estudio de estas aleaciones tiene mucho interés desde el punto de vista fundamental.

En este artículo se muestra el trabajo que se está llevando a cabo en el CENIM en este tipo de aleaciones.

\section{PARTE EXPERIMENTAL}

Las aleaciones estudiadas son:

- Al-Fe-Nb. Cinco aleaciones en torno a Al-9Fe$4 \mathrm{Nb}$ ( $\%$ at.), obtenidas en forma de cintas por temple ultrarrápido sobre la rueda.

- Al-5Cu-5Zr. Obtenidas en forma de polvos por atomización por gas inerte y por aleado mecánico y en forma de cintas por temple sobre rueda.

- Al-5Fe-5Nd y Al-5Fe-5Nd-5Nb obtenidas por aleado mecánico y temple sobre rueda.

La solidificación por temple sobre rueda consiste en proyectar la aleación fundida sobre una rueda que gira a gran velocidad, consiguiéndose velocidades de solidificación muy altas.

En la atomización por gas inerte, la aleación fundida es atomizada por chorros de gas a alta velocidad, solidificando y esferoidizando el material en vuelo.

El aleado mecánico, o molienda mecánica, es una técnica de deformación en frío que permite alear polvos metálicos mediante sucesivos mecanismos de deformación, fractura y soldadura que tienen lugar en un molino de bolas de alta energía, dando lugar a materiales con características similares a los solidificados rápidamente, pero dentro del estado sólido.
Las aleaciones así obtenidas se caracterizaron mediante difracción de rayos X (XRD), calorimetría diferencial de barrido (DSC), microscopía óptica, electrónica de transmisión y de barrido, y por medidas de microdureza.

\section{RESULTADOS Y DISCUSIÓN}

\subsection{Aleaciones Al-Fe-Nb}

La tabla I recoge las aleaciones estudiadas en este sistema y la figura 1 muestra los espectros de difracción de rayos $\mathrm{X}$ de las cintas, donde los halos presentes en las aleaciones 1 y 2 indican el carácter amorfo de estos materiales, mientras que las aleaciones 3,4 y 5 se presentan parcialmente amorfas,

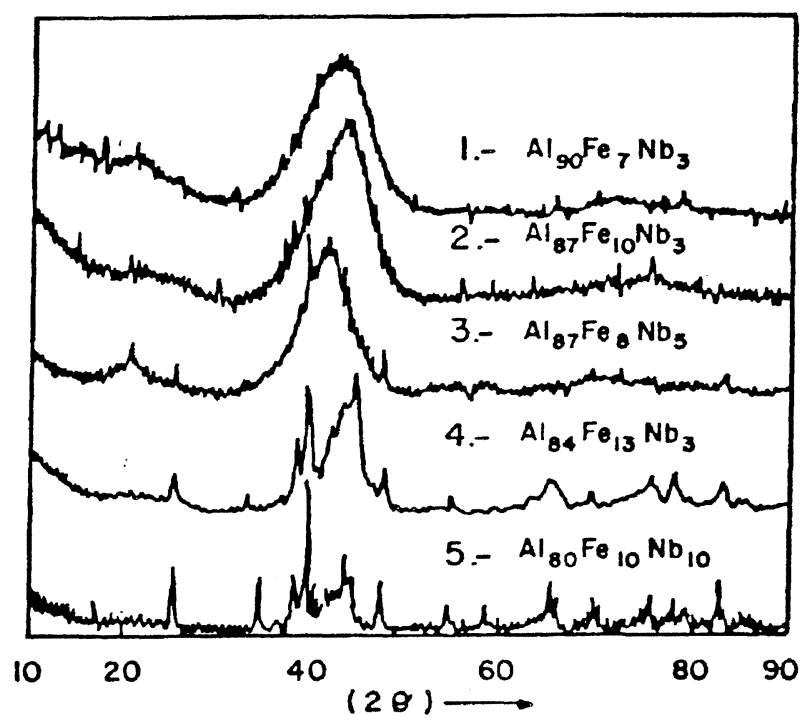

FIG. 1.- Espectros de difracción de rayos X de Al$\mathrm{Fe}-\mathrm{Nb}$.

FIG. 1.-Al-Fe-Nb X-ray diffraction patterns.

TABLA I.- Composición de las aleaciones (\% at.), estructura, temperatura del primer pico, $T_{\mathrm{p}}$, y del segundo, $T_{e}$, de cristalización, diferencia de temperatura entre ellos, $\Delta T$, para las aleaciones amorfas y microdureza Vickers $\left(\mathrm{kg} / \mathrm{mm}^{2}\right)$

TABLE I.- Alloy composition (at. \%), structure, first peak crystallization temperature, $\mathrm{T}_{\mathrm{p}}$, second peak crystallization temperature, $\mathrm{T}_{\mathrm{e}}$, of the amorphous alloys, temperature difference between both peaks, $\Delta \mathrm{T}$, and Vickers microhardness $\left(\mathrm{kg} / \mathrm{mm}^{2}\right)$

\begin{tabular}{|c|c|c|c|c|c|}
\hline Composición & Estructura & $\mathrm{Tp}$ & $\mathrm{Te}$ & $\Delta \mathrm{T}$ & $\mu \mathrm{Hv}$ \\
\hline $\mathrm{Al}_{90} \mathrm{Fe}_{7} \mathrm{Nb}_{3}$ & amorfa & $533 \mathrm{~K}$ & $693 \mathrm{~K}$ & $160 \mathrm{~K}$ & 300 \\
$\mathrm{Al}_{87} \mathrm{Fe}_{10} \mathrm{Nb}_{3}$ & amorfa & $623 \mathrm{~K}$ & $729 \mathrm{~K}$ & $106 \mathrm{~K}$ & 420 \\
$\mathrm{Al}_{87} \mathrm{Fe}_{8} \mathrm{Nb}_{5}$ & am. + cris. & & & & 470 \\
$\mathrm{Al}_{84} \mathrm{Fe}_{13} \mathrm{Nb}_{3}$ & am. + cris. & & & & 570 \\
$\mathrm{Al}_{80} \mathrm{Fe}_{10} \mathrm{Nb}_{10}$ & am. + cris. & & & & 550 \\
\hline
\end{tabular}


detectándose la presencia de fases cristalinas de equilibrio, $\mathrm{Al}$ c.c.c. $+\mathrm{Al}_{3} \mathrm{Fe}+\mathrm{Al}_{3} \mathrm{Nb}$.

La estabilidad térmica de las aleaciones amorfas fue estudiada por calorimetría diferencial de barrido (Fig. 2); se observan dos reacciones exotérmicas que indican que la cristalización de estas aleaciones tiene lugar en dos pasos: Una cristalización primaria, que da lugar a pequeños cristales de aluminio del orden de nanómetros, seguida de la cristalización eutéctica de la matriz amorfa remanente en $\mathrm{Al}_{3} \mathrm{Fe}$ y $\mathrm{Al}_{3} \mathrm{Nb}$.

Las medidas de microdureza Vickers (Tabla I) muestran altos valores en estos materiales, siendo más duras las aleaciones parcialmente cristalinas, pues la combinación de cristales y matriz amorfa endurece al material.

Resumiendo, se han obtenido aleaciones amorfas $\mathrm{Al}-\mathrm{Fe}-\mathrm{Nb}$, que al tratarlas térmicamente cristalizan primariamente dando lugar a nanocomposites formados por nanocristales de aluminio, embebidos en una matriz amorfa. La matriz remanente es muy estable, cristalizando $100 \mathrm{~K}$ por encima de los nanocristales de aluminio, lo que ofrece un amplio rango de estabilidad del nanocomposite. Su dureza es mayor que la de las aleaciones comerciales de aluminio y del mismo orden de las obtenidas por Inoue (8).

\subsection{Aleaciones $\mathrm{Al}-5 \mathrm{Cu}-5 \mathrm{Zr}$}

La aleación Al-5Cu-5Zr (\% at.), fue obtenida por tres procedimientos: temple sobre rueda, atomización por gas inerte y aleado mecánico.

Las cintas templadas sobre rueda son cristalinas y están compuestas por una solución sólida sobresaturada de aluminio y por pequeños precipitados de

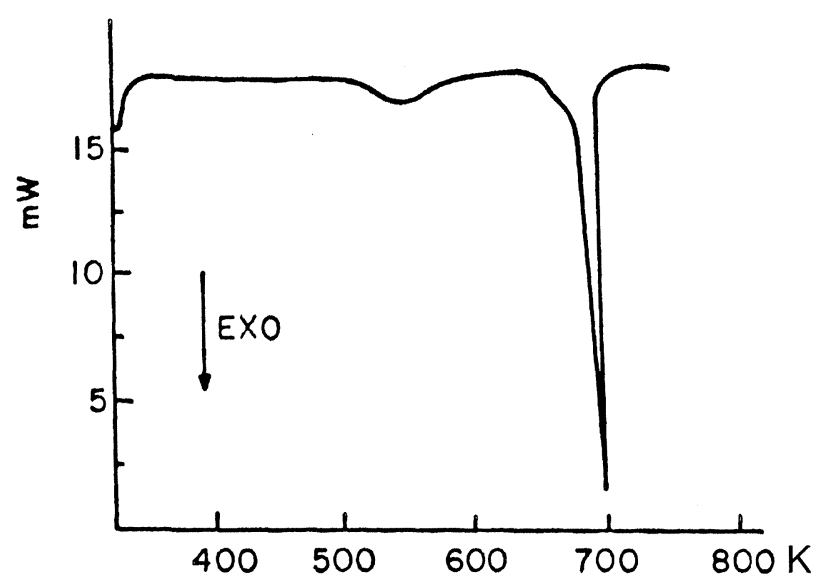

FIG. 2.- Calorimetría diferencial de barrido de la cinta amorfa $\mathrm{Al}_{90} \mathrm{Fe}_{7} \mathrm{Nb}_{3}$.

FIG. 2.-Differential scanning calorimeter trace of the $\mathrm{Al}_{90} \mathrm{Fe}_{7} \mathrm{Nb}_{3}$ amorphous ribbon.
$\mathrm{Al}_{2} \mathrm{Cu}$, del orden de $\mathrm{nm}$. Su microdureza (como recoge la tabla II) es alta y aumenta con los tratamientos térmicos hasta $285 \mathrm{~kg} / \mathrm{mm}^{2}$ al precipitar, a $673 \mathrm{~K}$, el circonio que estaba en solución sólida, y conservando aún a $773 \mathrm{~K}$ una dureza considerable.

El polvo atomizado no presenta solución sólida sobresaturada y su dureza, que es baja, sólo sube un poco tras el tratamiento a $673 \mathrm{~K}$. La tabla III presenta la estructura y la microdureza del polvo atomizado, así como tras los tratamientos térmicos de $1 \mathrm{~h}$ a distintas temperaturas.

Polvo atomizado de tamaño comprendido entre 100 y $200 \mu \mathrm{m}$ fue sometido a molienda mecánica en un molino de bolas de alta energía. La molienda dio lugar a la disolución de los compuestos de equilibrio, transformándose en una solución sólida sobresaturada que elevó la dureza del polvo, como se recoge en la tabla IV.

\subsection{Aleaciones Al-Fe-Nd}

Las aleaciones $\mathrm{Al}-5 \mathrm{Fe}-5 \mathrm{Nd}$ y $\mathrm{Al}-5 \mathrm{Fe}-5 \mathrm{Nd}-5 \mathrm{Nb}$ (\% at.), se han obtenido parcialmente amorfas por aleado mecánico a partir de los elementos puros. Al cristalizar, lo hacen en dos pasos y su microdureza está en torno a $280 \mathrm{~kg} / \mathrm{mm}^{2}$. Actualmente, se están obteniendo totalmente amorfas por temple ultrarrápido sobre la rueda y se están caracterizando con vistas a seleccionar la composición más adecuada para atomizar.

\section{CONCLUSIONES}

Se han obtenido aleaciones amorfas y nanoestructuradas de base aluminio a partir de procesos de temple ultrarrápido (sistema $\mathrm{Al}-\mathrm{Fe}-\mathrm{Nb}$ ) y molienda mecánica (aleaciones Al-Fe-Nd y Al-Fe-Nd-Nb). Las aleaciones $\mathrm{Al}-\mathrm{Fe}-\mathrm{Nb}$ presentan buenas características como material nanoestructurado y se prosigue su estudio, lo que constituirá la tesis de F. Audebert. La aleación $\mathrm{Al}-5 \mathrm{Cu}-5 \mathrm{Zr}$ no presenta buenas características para material nanoestructurado,

TABLA II.- Microestructura y microdureza Vickers de cintas $\mathrm{Al}-5 \mathrm{Cu}-5 \mathrm{Zr}$

TABLE II.- Microstructure and Vickers microhardness of melt-spun $\mathrm{Al}-5 \mathrm{Cu}-5 \mathrm{Zr}$ ribbons

\begin{tabular}{|c|c|c|}
\hline Tratamiento & Microestructura & $\mu \mathrm{Hv}, \mathrm{kg} / \mathrm{mm}^{2}$ \\
\hline Templada & $\mathrm{Al}+\mathrm{Al}_{2} \mathrm{Cu}$ & 255 \\
1 h a $573 \mathrm{~K}$ & $\mathrm{Al}+\mathrm{Al}_{2} \mathrm{Cu}$ & 245 \\
1 h a $673 \mathrm{~K}$ & $\mathrm{Al}+\mathrm{Al}_{2} \mathrm{Cu}$ & 285 \\
1 h a $773 \mathrm{~K}$ & $\mathrm{Al}+\mathrm{Al}_{2} \mathrm{Cu}+\mathrm{Al}_{3} \mathrm{Zr}$ & 145 \\
1 h a $923 \mathrm{~K}$ & $\mathrm{Al}+\mathrm{Al}_{3} \mathrm{Zr}+\mathrm{Al}_{2} \mathrm{Cu}$ & 76 \\
\hline
\end{tabular}

Rev. Metal. Madrid, 34 (mayo), $1998 \quad 251$ 
TABLA III.- Microestructura y microdureza Vickers de polvo atomizado $\mathrm{Al}-5 \mathrm{Cu}-5 \mathrm{Zr}$ de $50-100 \mu \mathrm{m}$ de diámetro

TABLE III.- Microstructure and Vickers microhardness of Al-5Cu-5Zr atomized powder, of 50-100 $\mu$ m diameter

\begin{tabular}{|c|c|c|}
\hline Tratamiento & Microestructura & $\mu \mathrm{Hv}, \mathrm{kg} / \mathrm{mm}^{2}$ \\
\hline Templado & $\mathrm{Al}+\mathrm{Al}_{2} \mathrm{Cu}+(\mathrm{Al}, \mathrm{Zr})$ & 81 \\
1 h a $573 \mathrm{~K}$ & $\mathrm{Al}+\mathrm{Al}_{2} \mathrm{Cu}+(\mathrm{Al}, \mathrm{Zr})$ & 72 \\
1 h a $673 \mathrm{~K}$ & $\mathrm{Al}+\mathrm{Al}_{2} \mathrm{Cu}+(\mathrm{Al}, \mathrm{Zr})$ & 88 \\
1 h a $773 \mathrm{~K}$ & $\mathrm{Al}+\mathrm{Al}_{2} \mathrm{Cu}+\mathrm{Al}_{3} \mathrm{Zr}$ & 81 \\
\hline
\end{tabular}

TABla IV.- Microestructura y microdureza Vickers de polvo atomizado $\mathrm{Al}-5 \mathrm{Cu}-5 \mathrm{Zr}$ y molido mecánicamente durante $140 \mathrm{~h}$

TABLE IV.- Microstructure and Vickers microhardness of Al-5Cu-5Zr atomized powder and mechanically milled for $140 \mathrm{~h}$

\begin{tabular}{|c|c|c|}
\hline Tratamiento & Microestructura & $\mu \mathrm{Hv}, \mathrm{kg} / \mathrm{mm}^{2}$ \\
\hline Molido & $\mathrm{Al}$ & 206 \\
1 h a $773 \mathrm{~K}$ & $\mathrm{Al}+\mathrm{Al}_{2} \mathrm{Cu}+\mathrm{Al}_{3} \mathrm{Zr}$ & 165 \\
\hline
\end{tabular}

pero el material molido mecánicamente tiene una buena estabilidad térmica, por lo que podría utilizarse como material para alta temperatura. El estudio de las aleaciones Al-Fe-Nd y Al-Fe-Nd-Nb, cuyo estudio consituirá la tesis de K. Cardoso, está aún en curso.

\section{Agradecimiento}

El presente Proyecto, MAT95-0796, ha sido financiado por la CICYT. Asimismo, K.R. Cardoso agradece la beca concedida por el Ministerio de Educación de Brasil y F. E. Audebert la beca MUTIS concedida por la Agencia Española de Cooperación Internacional, AECI. Los autores agradecen a M. Torralba, G. Caruana y M. Lieblich la atomización de la aleación Al- 5Cu-5Zr.

\section{REFERENCIAS}

(1) Masumoto, T. Mat. Sci. Eng. A179/180, 1994: 8.

(2) KIM, W.Y. Dispersion Strenghtened Aluminium Alloys, (Eds. Y.W.Kim y W.M.Griffith). TMS Publ., (PA., EE.UU.), 1988: 157.

(3) Palmer, I.G., Thomas, M.P. y Marshall, G.J. Dispersion Strenghtened Aluminum Alloys, (Eds. Y.W.Kim y W.M.Griffith), TMS Publ. (PA. EE.UU.), 1988: 217.

(4) Sanchez, B., Torralba, M., Cristina, M.C., Caruana, G. y GARCIA-EsCORIAL, A. Mat. Sci. Tech., 12, 1996: 794801.

(5) Lieblich, M., Caruana, G., Torralba, M. y Jones, H. Mat. Sci. Tech., 12, 1996 25-33.

(6) Inoue, A., Ohtera, K., Tsai, A.P. y Masumoto, T. Jpn. J. Appl. Phys. 27,1988: L280.

(7) He, Y., Poon, S.J. y Shiflet, G.J. Science 241, 1988: 1.640 .

(8) INoue, A. Mat. Sci. Eng. A179/A180, 1994: 8.

(9) Shechetman, D. y Blech, I.A. Metall. Trans. A16, 198: 1.005 .

(10) Audebert, F., Sirkin, H. y Garcia-Escorial, A. Scrip, Mat., 36, (4), 1997: 405.

(11) Audebert, F., Sirkin, H. y Garcia-Escorial, A. Phil. Mag. B, 76, (4), 1997: 483. 